\title{
On the number of irreducible polynomials with 0,1 coefficients
}

\author{
by
}

\section{S. V. Konyagin (Moscow)}

1. Introduction. For $d$ a positive integer, let

$$
\mathcal{P}_{d}=\left\{f(z): f(z)=\sum_{j=0}^{d} a_{j} z^{j}, a_{j}=0 \text { or } 1 \text { for all } j, a_{0}=a_{d}=1\right\} .
$$

By irreducibility we always mean irreducibility over the rationals. There is an intriguing conjecture that almost all polynomials $f(z)$ are irreducible, namely, the portion of irreducible polynomials in $\mathcal{P}_{d}$ tends to 1 as $d \rightarrow \infty$ (see [7]). This is still open. In what follows, $C, c$ will denote large and small absolute positive constants, respectively. In [2] it was proved that if $f(2)$ is prime for some $f \in \mathcal{P}_{d}$ then $f(z)$ is irreducible (see also [4]). Consequently, there are at least $c 2^{d} / d$ irreducible polynomials $f \in \mathcal{P}_{d}$. The same estimate can also be proved by calculation of polynomials $f \in \mathcal{P}_{d}$ irreducible over $\mathbb{F}_{2}$ [6, p. 93].

In this paper we improve the lower estimate of the number of irreducible polynomials of degree $d$ with 0,1 coefficients and establish

TheOREM 1. For a positive integer $d \geq 2$ there are at least $c 2^{d} / \log d$ irreducible polynomials $f \in \mathcal{P}_{d}$.

It is reasonable to conjecture that almost all reducible polynomials $f \in$ $\mathcal{P}_{d}$ are divisible by $z+1$; it would give the upper estimate $C 2^{d} / \sqrt{d}$ of the number of reducible polynomials $f \in \mathcal{P}_{d}$. We are able to prove this estimate of the number of polynomials with 0,1 coefficients possessing nontrivial divisors of small degree.

1991 Mathematics Subject Classification: Primary 11C08.

The author was supported by the National Science Foundation, grant DMS 9304580. This research was carried out while the author was a member of the Institute for Advanced Study, Princeton. It is a pleasure to thank the Institute for their hospitality and generosity. 
TheOREM 2. For a positive integer $d \geq 2$ and $m_{1}=[c d / \log d]$ there are at most $C 2^{d} / \sqrt{d}$ polynomials $f \in \mathcal{P}_{d}$ divisible by at least one integral polynomial of positive degree $\leq m_{1}$.

Theorem 1 follows easily from Theorem 2 . Indeed, consider the set $\Phi$ of integers $\gamma p \in\left[2^{d}, 2^{d+1}\right.$ ) where $p$ is prime, $\gamma$ is odd and $\gamma<\gamma_{1}=1.12^{m_{1}}$. For any $\gamma<\gamma_{1}$ the number of primes $p \in\left[2^{d} / \gamma, 2^{d+1} / \gamma\right)$, by the Prime Number Theorem, is

$$
\frac{2^{d}}{\gamma \log \left(2^{d} / \gamma\right)}(1+o(1)) \gg 2^{d} /(d \gamma)
$$

Hence,

$$
\# \Phi \gg \frac{2^{d}}{d} \sum_{\substack{\gamma<\gamma_{1} \\ \gamma \equiv 1(\bmod 2)}} \frac{1}{\gamma} \gg \frac{2^{d}}{d} \log \gamma_{1} \gg 2^{d} / \log d,
$$

i.e., ${ }^{\#} \Phi \geq c 2^{d} / \log d$. If $a_{d} \ldots a_{0}$ is the base 2 representation of an element $\varphi \in \Phi$, then the polynomial $f(z)=\lambda(\varphi)(z)=\sum_{j=0}^{d} a_{j} z^{j} \in \mathcal{P}_{d}$ and $f(2)$ $=\varphi$. Suppose that $f \in \lambda(\varphi)$ is reducible. Then $f=g_{1} g_{2}$ where $g_{1}, g_{2}$ are integral polynomials of positive degree and $\left|g_{1}(2)\right| \leq\left|g_{2}(2)\right|$. We have $\left|g_{1}(2)\right| \cdot\left|g_{2}(2)\right|=|f(2)|=\varphi$. If we assume that $g_{1}(2)$ is divisible by $p$, we get $\left|g_{2}(2)\right| \leq \gamma$ and

$$
\varphi=\left|g_{1}(2)\right| \cdot\left|g_{2}(2)\right| \leq\left|g_{2}(2)\right|^{2} \leq \gamma_{1}^{2}<2^{m_{1}}<\varphi .
$$

This contradiction shows that $g_{1}(2)$ cannot be divisible by $p$. Hence, $\left|g_{1}(2)\right|$ $\leq \gamma_{1}$.

To estimate the degree of $g_{1}$, we follow [2]. Let $m=\operatorname{deg} g_{1}, g_{1}(z)=$ $\pm \prod_{j=1}^{m}\left(z-z_{j}\right)$. Any $z_{j}$ is a zero of the polynomial $f$ with 0,1 coefficients. So, clearly, $\left|z_{j}\right|<2$, and, by [7], $\Re z_{j}<1.14$. These restrictions on $z_{j}$ imply $\left|z_{j}-2\right| /\left|z_{j}-1\right|>1.12$. Indeed, consider the function $V(z)=(z-1)^{2} /(z-2)^{2}$ on the closed domain $\Omega$ restricted by the segment of the line $\Re z=1.14,|\Im z|^{2}$ $\leq 4-1.14^{2}$, and the arc of the circle $|z|=2, \Re z \leq 1.14$. The maximum of $|V(z)|$ is attained on the boundary of $\Omega$. If $z$ is on the segment then

$$
\begin{aligned}
|V(z)| & =\left(0.14^{2}+\Im z^{2}\right) /\left(0.86^{2}+\Im z^{2}\right) \\
& \leq\left(0.14^{2}+4-1.14^{2}\right) /\left(0.86^{2}+4-1.14^{2}\right)=34 / 43 .
\end{aligned}
$$

If $z$ is on the arc then

$$
\begin{aligned}
|V(z)| & =\left(|z|^{2}+1-2 \Re z\right) /\left(|z|^{2}+4-4 \Re z\right)=(5-2 \Re z) /(8-4 \Re z) \\
& \leq(5-2 \cdot 1.14) /(8-4 \cdot 1.14)=34 / 43 .
\end{aligned}
$$

Hence, for any $z \in \Omega$ the inequality $V(z) \leq 34 / 43$ holds, and for any zero $z_{j}$ of the polynomial $g_{1}$ we have

$$
\left|z_{j}-2\right| /\left|z_{j}-1\right|=\left|V\left(z_{j}\right)\right|^{-1 / 2} \geq(34 / 43)^{-1 / 2}>1.12 .
$$


Therefore,

$$
1.12^{m_{1}}=\gamma_{1} \geq\left|g_{1}(2)\right| /\left|g_{1}(1)\right| \geq 1.12^{m},
$$

and $\operatorname{deg} g_{1}=m \leq m_{1}$. By Theorem 2 , there are at most $C 2^{d} / \sqrt{d}$ reducible polynomials in the set $\lambda(\Phi)$, but this set contains at least $c 2^{d} / \log d$ elements. Thus, $\lambda(\Phi)$ contains as many irreducible polynomials as required in Theorem 1.

Throughout the following we assume that the number $d$ is sufficiently large.

Denote by $\mathcal{D}$ the set of polynomials

$$
g(z)=\sum_{j=0}^{m} b_{j} z^{j}, \quad b_{m}=1, \quad m \in \mathbb{N},
$$

with integral coefficients. To prove Theorem 2, we will estimate the number of polynomials $f \in \mathcal{P}_{d}$ divisible by an irreducible polynomial $g \in \mathcal{D}$, and then take the sum over all polynomials $g$ of degree at most $m_{1}$. To motivate our proof of Theorem 2, we indicate how to obtain a weaker result: Theorem 2 is valid if we replace $m_{1}$ by $m_{2}=\left[\sqrt{d} /(\log d)^{2}\right]$. For any polynomial $g$ with leading coefficient \pm 1 we define

$$
M(g)=\prod_{j=1}^{m} \max \left(1,\left|z_{j}\right|\right),
$$

where $z_{1}, \ldots, z_{m}$ are the zeros of $g$ counted with multiplicity. By Jensen's theorem (Theorem 3.61 of [10]),

$$
\log M(g)=\frac{1}{2 \pi} \int_{0}^{2 \pi} \log \left|g\left(e^{i \varphi}\right)\right| d \varphi
$$

Therefore,

$$
M\left(\sum_{j=0}^{m} b_{j} z^{j}\right) \leq \sum_{j=0}^{m}\left|b_{j}\right| .
$$

Clearly, $M(g) \geq 1$, and Kronecker's theorem [5] asserts that $M(g)=1$ implies that all zeros of $g$ are roots of unity. Otherwise, as was proved by Dobrowolski [3],

$$
M(g) \geq \exp \left(\lambda_{m}\right), \quad \lambda_{m}=c\left(\frac{\log \log m}{\log m}\right)^{3} \quad(m \geq 3) .
$$

Take an arbitrary noncyclotomic irreducible polynomial $g \in \mathcal{D}$ such that $\operatorname{deg} g=m \leq m_{2}$, and let $z_{1}, \ldots, z_{m}$ be the zeros of $g$. By Lemma 3 of [3], there exists a prime $p$ such that $\log (d+1) / \lambda_{m_{2}}<p<2 \log (d+1) / \lambda_{m_{2}}$ and all $z_{j}^{p}$ are algebraic numbers of degree $m$ (and, therefore, are distinct). Set 
$g_{p}(w)=\prod_{j=1}^{m}\left(w-z_{j}^{p}\right)$. Then, taking into account (1.2), we get

$$
M\left(g_{p}\right)=M(g)^{p} \geq \exp \left(p \lambda_{m_{2}}\right)>d+1 .
$$

Suppose that $g(z)$ divides two distinct polynomials $f_{1}$ and $f_{2}$ from $\mathcal{P}_{d}$ such that

$$
f_{1}(z)-f_{2}(z)=\sum_{j=0}^{[d / p]} a_{j} z^{j p}=h\left(z^{p}\right) .
$$

Clearly, all coefficients of the polynomial $h$ are $0, \pm 1$. By $(1.1), M(h) \leq d+1$. On the other hand, any zero $z_{j}^{p}$ of $g_{p}$ is a zero of $h$. Hence, $h$ is divisible by $g_{p}$, and (1.3) entails $M(h)>d+1$. This contradiction shows that our supposition cannot occur. This means that if a polynomial $f(z)=\sum_{j=0}^{d} a_{j} z^{j} \in \mathcal{P}_{d}$ is divisible by $g$ then $f$ is uniquely determined by its coefficients $a_{j}, j \not \equiv 0$ $(\bmod p)$. Hence,

$$
\begin{aligned}
\#\left\{f \in \mathcal{P}_{d}: g \mid f\right\} & <2^{d} / 2^{d / p}<2^{d} / 2^{d \lambda_{m_{2}} /(2 \log (d+1))} \\
& <2^{d} \exp \left(-0.3 d \lambda_{m_{2}} / \log d\right) .
\end{aligned}
$$

Let us estimate the number $N$ of polynomials $g \in \mathcal{D}$ of degree at most $m_{2}$ dividing at least one polynomial $f \in \mathcal{P}_{d}$. We consider any such polynomial

$$
g(z)=\sum_{j=0}^{m} b_{j} z^{j}=\prod_{j=1}^{m}\left(z-z_{j}\right) .
$$

Since $\left|z_{j}\right|<2$ for any $j$, representing the coefficients of the polynomial $g$ as symmetric polynomials of its zeros, we find $\left|b_{j}\right| \leq 2^{m-j}\left(\begin{array}{c}m \\ j\end{array}\right)<4^{m}$. Hence,

$$
N<\prod_{j=0}^{m_{2}}\left(2 \cdot 4^{m_{2}}+1\right)<5^{m_{2}^{2}} .
$$

It follows from the last inequality and (1.4) that the total number of polynomials $f \in \mathcal{P}_{d}$ divisible by at least one noncyclotomic polynomial of degree at most $m_{2}$ is less than

$$
\begin{aligned}
2^{d} 5^{m_{2}^{2}} \exp \left(-0.3 d \lambda_{m_{2}} / \log d\right) & <2^{d} \exp \left(2 d /(\log d)^{4}-0.3 d \lambda_{m_{2}} / \log d\right) \\
& =o\left(2^{d} / \sqrt{d}\right) .
\end{aligned}
$$

It is not difficult to prove that the number of polynomials $f \in \mathcal{P}_{d}$ divisible by at least one cyclotomic polynomial is $O\left(2^{d} / \sqrt{d}\right)$ (see $\S 4$ ). Thus, we get the analog of Theorem 2 for $m_{2}$ instead of $m_{1}$.

To prove Theorem 2, we must have more accurate estimates for the number of possible divisors of polynomials $f \in \mathcal{P}_{d}$. In Section 3 we will find upper bounds for the number of polynomials $g \in \mathcal{D}$ with restrictions on their zeros. To do this, we need some estimates of $\varepsilon$-capacity of appropriate 
convex bodies in finite-dimensional spaces. Such estimates will be found in Section 2. Theorem 2 will be proved in Section 4 .

2. Several geometric lemmas. In this section we fix two positive numbers $\alpha$ and $\tau$ such that

$$
\alpha>1, \quad \tau<0.1 / \alpha .
$$

Also, $m$ will denote a sufficiently large positive integer exceeding some magnitude depending on $\alpha$ and $\tau$. Let $X=X_{m}$ be the $m$-dimensional real coordinate space equipped with the $l_{\infty}$-norm:

$$
\left|\left(x_{1}, \ldots, x_{m}\right)\right|=\max _{1 \leq j \leq m}\left|x_{j}\right| .
$$

We denote by $\operatorname{Vol}(A)=\operatorname{Vol}_{m}(A)$ the volume of a convex closed bounded set $A \subset X$.

Lemma 2.1. Let $\Pi \subset \mathbb{R}^{l}$ be a parallelepiped and $A \subset \Pi$ be a convex polytope with $n \leq l^{\alpha}$ vertices. Then

$$
\operatorname{Vol}(A)<\operatorname{Vol}(\Pi)\left(\frac{90 \alpha \log l}{l}\right)^{l / 2},
$$

provided that $l$ is large enough.

Proof. We may suppose that $\Pi$ is a cube inscribed in the unit ball $B$, namely, $\Pi=[-1 / \sqrt{l}, 1 / \sqrt{l}]^{l}$. We have

$$
\operatorname{Vol}(B)=\frac{\pi^{l / 2}}{\Gamma(1+l / 2)}<\frac{\pi^{l / 2}}{(l /(2 e))^{l / 2}}=\left(\frac{2 \pi e}{l}\right)^{l / 2}=(\pi e)^{l / 2} \operatorname{Vol}(\Pi) .
$$

Using the inequality

$$
\operatorname{Vol}(A) \leq \operatorname{Vol}(B)\left(\frac{10 \alpha \log l}{l}\right)^{l / 2}
$$

for the volume of a convex polytope $A$ with $\leq l^{\alpha}$ vertices provided that $l>l(\alpha)$ (see [1]) we get

$$
\operatorname{Vol}(A) \leq \operatorname{Vol}(\Pi)\left(\frac{10 \pi e \alpha \log l}{l}\right)^{l / 2}<\left(\frac{90 \alpha \log l}{l}\right)^{l / 2},
$$

as required.

Let $e_{1}, \ldots, e_{m}$ be the coordinate vectors of $X_{m}$. For a convex closed bounded set $A \subset X$ and $j=1, \ldots, m$ define

$$
O_{j}(A)=A+\left[-e_{j} / 2, e_{j} / 2\right]=\left\{x+\mu e_{j}: x \in A,|\mu| \leq 1 / 2\right\} .
$$

Let $O(A)$ be the $1 / 2$-neighborhood of $A$ :

$$
O(A)=\{x+y: x \in A,|y| \leq 1 / 2\} .
$$


We have

$$
O(A)=O_{1}\left(\ldots O_{m}(A) \ldots\right) .
$$

Let $\Sigma=\Sigma(m)$ be the set of all subsets of the set $\{1, \ldots, m\}$ and $T \in \Sigma$. We denote by $P_{T}(A)$ the orthogonal projection of the set $A$ to the linear space spanned by $e_{j}, j \in T$. We consider that $\operatorname{Vol}_{0}\left(P_{\emptyset}(A)\right)=1$.

Lemma 2.2. For any $T \in \Sigma$ and any $j \in T$ we have

$$
\operatorname{Vol}_{\#_{T}}\left(P_{T}\left(O_{j}(A)\right)\right)=\operatorname{Vol}_{\#_{T}}\left(P_{T}(A)\right)+\operatorname{Vol}_{\# T-1}\left(P_{T \backslash\{j\}}(A)\right) .
$$

Proof. Set $T^{\prime}=T \backslash\{j\}$ and represent $P_{T}$ in the following form:

$$
\begin{aligned}
P_{T}(A)=\left\{\left\{x_{t}: t \in T\right\}:\left\{x_{t}: t \in T^{\prime}\right\} \in P_{T^{\prime}}(A),\right. & \\
& \left.f_{1}\left(\left\{x_{t}: t \in T^{\prime}\right\}\right) \leq x_{j} \leq f_{2}\left(\left\{x_{t}: t \in T^{\prime}\right\}\right)\right\} .
\end{aligned}
$$

Then

$$
\begin{aligned}
P_{T}\left(O_{j}(A)\right)=\{ & \left\{x_{t}: t \in T\right\}:\left\{x_{t}: t \in T^{\prime}\right\} \in P_{T^{\prime}}(A), \\
& \left.f_{1}\left(\left\{x_{t}: t \in T^{\prime}\right\}\right)-1 / 2 \leq x_{j} \leq f_{2}\left(\left\{x_{t}: t \in T^{\prime}\right\}\right)+1 / 2\right\} .
\end{aligned}
$$

Expressing the volumes of $P_{T}(A)$ and $P_{T}\left(O_{j}(A)\right)$ as integrals over $P_{T^{\prime}}(A)$ we get the required relationship. Lemma 2.2 is proved.

Using (2.2), we can write

$$
O(A)=P_{\{1, \ldots, m\}}\left(O_{1}\left(\ldots O_{m}(A) \ldots\right) .\right.
$$

Now, applying subsequently Lemma 2.2 , and taking into account that the operators $T$ and $O_{j}$ commute for $j \in T$, we obtain

Lemma 2.3. The following equality holds:

$$
\operatorname{Vol}(O(A))=\sum_{T \in \Sigma} \operatorname{Vol}_{\# T}\left(P_{T}(A)\right) .
$$

In the next lemma we estimate the $1 / 2$-capacity $[9,1.1 .7]$ of convex polytopes contained in a parallelepiped.

Lemma 2.4. Let $\Pi=\prod_{j=1}^{m}\left[-u_{j} / 2, u_{j} / 2\right]$ be a parallelepiped and $A \subset \Pi$ be a convex polytope with $n \leq m^{\alpha-1}$ vertices. Let $D$ be a subset of $A$ such that the distance between any two elements of $D$ is at least 1 . Then

$$
{ }^{\#} D<\exp \left(m^{1-9 \tau}\right) \prod_{j=1}^{m}\left(1+\frac{u_{j}}{m^{0.5-6 \tau}}\right) .
$$

Proof. The unit cubes with centers at the points of $D$ are mutually nonoverlapping, and the union of these cubes is a subset of $O(A)$. Therefore, ${ }^{\#} D \leq \operatorname{Vol}(O(a))$, and it remains to estimate the volume of $O(A)$.

We use Lemma 2.3. Note that in view of the inequality (2.1), $n<$ $\left(m^{1-10 \tau}\right)^{\alpha}$. Therefore, for $l={ }^{\#} T \geq m^{1-10 \tau}$ the volumes on the right-hand 
side of (2.3) can be estimated by Lemma 2.1:

$$
\begin{aligned}
\operatorname{Vol}_{l}\left(P_{T}(A)\right) & <\operatorname{Vol}_{l}\left(P_{T}(\Pi)\right)\left(\frac{90 \alpha \log l}{l}\right)^{l / 2} \\
& \leq \operatorname{Vol}_{l}\left(P_{T}(\Pi)\right)\left(\frac{90 \alpha \log m}{m^{1-10 \tau}}\right)^{l / 2}<\operatorname{Vol}_{l}\left(P_{T}(\Pi)\right)\left(m^{0.5-6 \tau}\right)^{-l} .
\end{aligned}
$$

For $l<m^{1-10 \tau}$ using the trivial estimate $\operatorname{Vol}_{l}\left(P_{T}(A)\right) \leq \operatorname{Vol}_{l}\left(P_{T}(\Pi)\right)$ we have

$$
\begin{aligned}
\operatorname{Vol}_{l}\left(P_{T}(A)\right) & \leq \operatorname{Vol}_{l}\left(P_{T}(\Pi)\right)\left(m^{0.5-6 \tau}\right)^{-l}\left(m^{0.5-6 \tau}\right)^{l} \\
& <\operatorname{Vol}_{l}\left(P_{T}(\Pi)\right)\left(m^{0.5-6 \tau}\right)^{-l}\left(m^{0.5-6 \tau}\right)^{m^{1-10 \tau}} \\
& <\operatorname{Vol}_{l}\left(P_{T}(\Pi)\right)\left(m^{0.5-6 \tau}\right)^{-l} \exp \left(m^{1-9 \tau}\right) .
\end{aligned}
$$

In both cases we have the inequality

$$
\operatorname{Vol}_{\#_{T}}\left(P_{T}(A)\right)<\operatorname{Vol}_{\#_{T}}\left(P_{T}(\Pi)\right)\left(m^{0.5-6 \tau}\right)^{-{ }^{\#} T} \exp \left(m^{1-9 \tau}\right) .
$$

Now, using (2.3), we find

$$
\begin{aligned}
\operatorname{Vol}(O(A)) & <\exp \left(m^{1-9 \tau}\right) \sum_{T \in \Sigma} \operatorname{Vol}_{\#_{T}}\left(P_{T}(\Pi)\right)\left(m^{0.5-6 \tau}\right)^{-{ }^{\#} T} \\
& =\exp \left(m^{1-9 \tau}\right) \prod_{j=1}^{m}\left(1+\frac{u_{j}}{m^{0.5-6 \tau}}\right)
\end{aligned}
$$

This completes the proof of Lemma 2.4.

The following lemma is the only statement of this section which will be used later on.

Lemma 2.5. For a vector $w=\left(w_{1}, \ldots, w_{m}\right) \in \mathbb{C}^{m}$ define

$$
S_{k}(w)=\sum_{j=1}^{m} w_{j}^{k} \quad(k=1, \ldots, m) .
$$

Let $W$ be a subset of $\mathbb{C}^{m}$ such that $\max _{j}\left|w_{j}\right| \leq \exp (0.4(\log m) / m)$ for any $w=\left(w_{1}, \ldots, w_{m}\right) \in W$ and $\max _{1 \leq k \leq m}\left|\Re S_{k}(w)-\Re S_{k}\left(w^{\prime}\right)\right| / k>1 / 2$ for any two distinct vectors $w, w^{\prime}$ from $W$. Then ${ }^{\#} W<\exp \left(m^{0.95}\right)$.

Proof. We associate with any vector $w \in W$ the vector $\widetilde{w}=\left(\widetilde{w}_{1}, \ldots\right.$ $\left.\ldots, \widetilde{w}_{m}\right)$ such that

$$
\begin{array}{r}
\widetilde{w}_{j}=m^{-2.45}\left(\operatorname{sgn}\left(\Re w_{j}\right)\left[m^{2.45}\left|\Re w_{j}\right|\right]+i \operatorname{sgn}\left(\Im w_{j}\right)\left[m^{2.45}\left|\Im w_{j}\right|\right]\right) \\
\quad(j=1, \ldots, m),
\end{array}
$$

where $[\cdot]$ denotes the integral part. For any $j \in\{1, \ldots, m\}$ and $k \in\{1, \ldots, m\}$ 
we have $\left|w_{j}-\widetilde{w}_{j}\right| \leq \sqrt{2} m^{-2.45}$ and

$$
\begin{aligned}
\left|w_{j}^{k}-\widetilde{w}_{j}^{k}\right| & \leq k\left|w_{j}-\widetilde{w}_{j}\right| \max \left(\left|w_{j}\right|^{k-1},\left|\widetilde{w}_{j}\right|^{k-1}\right) \\
& \leq 2 k m^{-2.45}(\exp (0.4(\log m) / m))^{k-1} \\
& \leq 2 m^{-1.45}(\exp (0.4(\log m) / m))^{m}=2 m^{-1.05} .
\end{aligned}
$$

From the last inequality and the assumption on $W$ we get

$$
\max _{1 \leq k \leq m}\left|\Re S_{k}(\widetilde{w})-\Re S_{k}\left(\widetilde{w}^{\prime}\right)\right| / k>1 / 3 \quad\left(w, w^{\prime} \in W, w \neq w^{\prime}\right) .
$$

Consider the mapping $\psi: W \rightarrow X$ :

$$
\psi(w)=\left(3 \Re S_{1}(\widetilde{w}), 3 \Re S_{2}(\widetilde{w}) / 2, \ldots, 3 \Re S_{m}(\widetilde{w}) / m\right) .
$$

The condition (2.5) means that all distances between $\psi(w)$ and $\psi\left(w^{\prime}\right)$ for distinct $w, w^{\prime} \in W$ are at least 1 .

The set $D=\psi(W)$ is contained in the convex hull $A$ of the vectors $\left(3 m \Re z, 3 m \Re\left(z^{2}\right) / 2, \ldots, 3 m \Re\left(z^{m}\right) / m\right)$, where $|z| \leq \exp (0.4(\log m) / m)$ and $m^{2.45} \Re z, m^{2.45} \Im z$ are integers (i.e. $z$ runs over the set of all possible points $\left.\widetilde{w}_{j}\right)$. The number $n$ of the vertices of the polytope $A$ does not exceed

$$
\pi\left(1+\exp (0.4(\log m) / m) / m^{-2.45}\right)^{2}<m^{6} .
$$

Moreover, $A$ is contained in the parallelepiped

$$
\Pi=\prod_{j=1}^{m}\left[-u_{j} / 2, u_{j} / 2\right], \quad u_{j}=6 m \exp (0.4(j / m) \log m) / j \quad(j=1, \ldots, m) .
$$

Now we are ready to apply Lemma 2.4. Take $\alpha=7, \tau=1 / 150$. Lemma 2.4 asserts that

$$
{ }^{\#} D<\exp \left(m^{0.94}\right) \prod_{j=1}^{m}\left(1+\frac{u_{j}}{m^{0.46}}\right) .
$$

We have

$$
\log \prod_{j=1}^{m}\left(1+\frac{u_{j}}{m^{0.46}}\right) \leq \sum_{j=1}^{m} \frac{u_{j}}{m^{0.46}} \leq \sum_{j=1}^{m} \frac{6 m^{0.94}}{j}<m^{0.945} .
$$

Substitution of the last inequality into (2.6) implies

$$
{ }^{\#} D<\exp \left(m^{0.94}\right) \exp \left(m^{0.945}\right)<\exp \left(m^{0.95}\right) .
$$

But ${ }^{*} W={ }^{*} D$. Thus, ${ }^{\#} W<\exp \left(m^{0.95}\right)$, as required.

3. Estimates of the number of irreducible polynomials with restrictions on its zeros. Let $g \in \mathcal{D}$ be an irreducible polynomial and $z$ be one of its zeros. For an integer $l$ we denote the number of zeros $z^{\prime}$ of the polynomial $g$ such that $\left(z^{\prime}\right)^{l}=z^{l}$ by $k_{l}(g)$. Clearly, $k_{l}(g)$ does not depend on the 
choice of $z$. Moreover, $k_{l}(g)$ divides the degree of $g$. For a nonnegative number $U$ and positive integers $k, l, m, m \geq 2$, we will denote by $\mathcal{D}(U, m, l, k)$ the set of irreducible polynomials $g$ of degree $m$ such that $\log M(g) \leq U / m$ and $k_{l}(g)=k$. We consider that $k$ divides $m$ since otherwise the set $\mathcal{D}(U, m, l, k)$ is empty.

LEMMA 3.1. For sufficiently large $m$ the cardinality of $\mathcal{D}(U, m, l, k)$ does not exceed

$$
\exp \left(m^{0.95}\right) C^{U / k}(C l / k)^{C U / \log m} .
$$

REMARK. In the case $l=1$ the proof of the lemma actually shows that the number of all polynomials $g(z)=z^{m}+\sum_{j=0}^{m-1} b_{j} z^{j}$ with integral coefficients such that $\log M(g) \leq U / m$ does not exceed $\exp \left(m^{0.95}\right) C^{U}$.

Pr o of (of Lemma 3.1). We say that the zeros $v, v^{\prime}$ of the polynomial $g \in$ $\mathcal{D}(U, m, l, k)$ are equivalent if $v^{l}=\left(v^{\prime}\right)^{l}$. Fix a maximal subset $\left\{v_{1}, \ldots, v_{m / k}\right\}$ of mutually nonequivalent zeros of $g$ such that $\left|v_{1}\right| \geq \ldots \geq\left|v_{m / k}\right|$. Let $\left|v_{n}\right| \geq \exp (0.4(\log m) / m)>\left|v_{n+1}\right|$ (for definiteness, we consider $v_{0}=\infty$, $\left.v_{m / k+1}=0\right)$. Define $n=\psi(g)$. Besides the mapping $\psi$, we define several mappings on the set $\mathcal{D}(U, m, l, k)$. Let $\psi_{j}(g)=\left[m \log \left|v_{j}\right|\right](j=1, \ldots, n)$. We have

$$
\prod_{j=1}^{n}\left|v_{j}\right| \leq \prod_{j=1}^{m / k} \max \left(1,\left|v_{j}\right|\right)=(M(g))^{1 / k} \leq \exp (U /(m k)) .
$$

On the other hand,

$$
\prod_{j=1}^{n}\left|v_{j}\right| \geq \exp (0.4 n(\log m) / m)
$$

and

$$
\prod_{j=1}^{n}\left|v_{j}\right| \geq \prod_{j=1}^{n} \exp \left(\psi_{j}(g) / m\right)=\exp \left(\sum_{j=1}^{n} \psi_{j}(g) / m\right) .
$$

Therefore,

$$
\psi(g)=n \leq U /(0.4 k \log m)
$$

and

$$
\sum_{j=1}^{n} \psi_{j}(g) \leq U / k
$$

For any $u \in \mathbb{Z}_{+}$we cover the disk $\{z:|z| \leq \exp ((u+1) / m)\}$ by disjoint squares

$$
\begin{aligned}
S_{\nu}^{u} & =\left[\alpha /\left(m^{3} v\right),(\alpha+1) /\left(m^{3} v\right)\right) \times\left[\beta /\left(m^{3} v\right),(\beta+1) /\left(m^{3} v\right)\right), \\
v & =\exp ((m-1) u / m), \quad \alpha, \beta \in \mathbb{Z}, \quad \nu \in \mathbb{Z}, 1 \leq \nu \leq N_{u} .
\end{aligned}
$$


Taking into account that

$$
v \exp ((m-1) u / m)=\exp (u+1 / m)<3 \exp (u),
$$

we can write a rough estimate for the number $N_{u}$ of squares intersecting the disk:

$$
N_{u} \leq m^{7} \exp (2 u)
$$

We define the mappings $\Psi_{j}(j=1, \ldots, n)$ setting $\Psi_{j}(g)=\nu$ if $v_{j} \in S_{\nu}^{u}$ where $u=\psi_{j}(g)$. Finally, for $j=1, \ldots, n$ we define the mapping $\varphi_{j}$ from $\mathcal{D}(U, m, l, k)$ to the set $\Sigma(k, l)$ of $k$-element subsets of $\{1, \ldots, l\}$ by setting $\varphi_{j}(g)=T \in \Sigma(k, l)$ if the set of zeros of $g$ equivalent to $v_{j}$ is the set of numbers $v_{j} \zeta^{t}, t \in T$, where $\zeta$ is a fixed primitive $l$ th root of unity.

We want to estimate the number of distinct images

$$
\left(n=\psi(g), \psi_{1}(g), \ldots, \psi_{n}(g), \Psi_{1}(g), \ldots, \Psi_{n}(g), \varphi_{1}(g), \ldots, \varphi_{n}(g)\right)
$$

for $g \in \mathcal{D}(U, m, l, k)$.

For a fixed $n=\psi(g)$ the values $\psi_{1}(g), \ldots, \psi_{n}(g)$ are determined by $n$ different numbers $\psi_{1}(g), \psi_{1}(g)+\psi_{2}(g), \ldots, \psi_{1}(g)+\ldots+\psi_{n}(g)$ not exceeding $[U / k]$ by (3.2). Consequently,

$$
\#\left\{\left(n, \psi_{1}(g), \ldots, \psi_{n}(g)\right)\right\} \leq \sum_{n=0}^{[U / k]}\left(\begin{array}{c}
{[U / k]} \\
n
\end{array}\right) \leq 2^{U / k} .
$$

Then, for fixed $n, \psi_{1}(g), \ldots, \psi_{n}(g)$ we have, by (3.3), (3.1) and (3.2),

$$
\begin{aligned}
\#\left\{\left(\Psi_{1}(g), \ldots, \Psi_{n}(g)\right)\right\} & \leq \prod_{j=1}^{n}\left(m^{7} \exp \left(2 \psi_{j}(g)\right)\right) \\
& \leq m^{7 U /(0.4 k \log m)} \exp (2 U / k) \leq C^{U / k} .
\end{aligned}
$$

Finally, for fixed $n, \psi_{1}(g), \ldots, \psi_{n}(g), \Psi_{1}(g), \ldots, \Psi_{n}(g)$, using (3.1) again and the inequality

$$
\left(\begin{array}{l}
l \\
k
\end{array}\right) \leq l^{k} / k ! \leq(e l / k)^{k}
$$

we obtain

$$
\begin{aligned}
& { }^{\#}\left\{\left(\varphi_{1}(g), \ldots, \varphi_{n}(g)\right)\right\} \\
& \quad \leq\left(\begin{array}{l}
l \\
k
\end{array}\right)^{n} \leq(e l / k)^{k U /(0.4 k \log m)} \leq(e l / k)^{C U / \log m} .
\end{aligned}
$$

The combination of inequalities (3.4)-(3.6) implies

$$
\begin{aligned}
\#\left\{\left(n, \psi_{1}(g), \ldots, \psi_{n}(g), \Psi_{1}(g), \ldots,\right.\right. & \left.\left.\Psi_{n}(g), \varphi_{1}(g), \ldots, \varphi_{n}(g)\right)\right\} \\
& \leq N=(2 C)^{U / k}(e l / k)^{C U / \log m} .
\end{aligned}
$$



that

Consider two polynomials $g \in \mathcal{D}(U, m, l, k)$ and $\widetilde{g} \in \mathcal{D}(U, m, l, k)$ such

$$
\begin{gathered}
\psi(g)=\psi(\widetilde{g}), \quad \psi_{j}(g)=\psi_{j}(\widetilde{g}) \quad(j=1, \ldots, n), \\
\Psi_{j}(g)=\Psi_{j}(\widetilde{g}) \quad(j=1, \ldots, n), \quad \varphi_{j}(g)=\varphi_{j}(\widetilde{g}) \quad(j=1, \ldots, n) .
\end{gathered}
$$

Define

$$
\begin{aligned}
Z & =\{z: g(z)=0\}, \quad \widetilde{Z}=\{z: \widetilde{g}(z)=0\}, \\
V & =\{v \in Z:|v| \geq \exp (0.4(\log m) / m)\}, \\
\widetilde{V} & =\{v \in \widetilde{Z}:|v| \geq \exp (0.4(\log m) / m)\}, \\
W & =\{w \in Z:|w|<\exp (0.4(\log m) / m)\}, \\
\widetilde{W} & =\{w \in \widetilde{Z}:|w|<\exp (0.4(\log m) / m)\} .
\end{aligned}
$$

Let $n=\psi(g)=\psi(\widetilde{g})$. For any $j=1, \ldots, n$ we take $u=\psi_{j}(g)=\psi_{j}(\widetilde{g})$, $v=\exp ((m-1) u / m)$ and the corresponding zeros $v_{j}$ of $g$ and $\widetilde{v}_{j}$ of $\widetilde{g}$. Since the values of $\Psi_{j}$ at $g$ and $\widetilde{g}$ coincide, we have

$$
\begin{aligned}
\left|v_{j}-\widetilde{v}_{j}\right| & \leq \sqrt{2} /\left(m^{3} v\right)<4 /\left(m^{3} \exp ((m-1)(u+1) / m)\right) \\
& \leq 4 \max \left(\left|v_{j}\right|,\left|\widetilde{v}_{j}\right|\right)^{1-m} / m^{3} .
\end{aligned}
$$

For any zero $v \in V$ of $g$ equivalent to $v_{j}$ we set $\chi(v)=v \widetilde{v}_{j} / v_{j}$. As the values of $\varphi_{j}$ at $g$ and $\widetilde{g}$ coincide, $\chi$ is a one-to-one correspondence $V \rightarrow \widetilde{V}$. By (3.9), $|\chi(v)-v| \leq 4\left|\max (|v|,|\chi(v)|)^{1-m}\right| / m^{3}$ for any $v \in V$. Therefore,

$$
\begin{aligned}
\left|(\chi(v))^{i}-v^{i}\right| & \leq i \max (|v|,|\chi(v)|)^{i-1}|\chi(v)-v| \\
& <m \max (|v|,|\chi(v)|)^{m-1}|\chi(v)-v| \leq 4 / m^{2} \quad(i=1, \ldots, m),
\end{aligned}
$$

and

$$
\left|\sum_{v \in V} v^{i}-\sum_{v \in \widetilde{V}} v^{i}\right|<1 / 2 \quad(i=1, \ldots, m)
$$

Let

$$
\begin{gathered}
g(z)=\sum_{j=0}^{m} b_{j} z^{j}, \quad b_{m}=1, \\
S_{i}=\sum_{z \in Z} z^{i}=\sum_{v \in V} v^{i}+\sum_{w \in W} w^{i} \quad(i=1, \ldots, m), \\
\widetilde{S}_{i}=\sum_{z \in \widetilde{Z}} z^{i}=\sum_{v \in \widetilde{V}} v^{i}+\sum_{w \in \widetilde{W}} w^{i} \quad(i=1, \ldots, m) .
\end{gathered}
$$

The numbers $S_{1}, \ldots, S_{m}$ are integers. Moreover, by the Newton identities,

$$
S_{i}+b_{m-1} S_{i-1}+\ldots+b_{m-i+1} S_{1}+i b_{m-i}=0 \quad(i=1, \ldots, m) .
$$


Therefore, $S_{1}, \ldots, S_{i-1}$ determine $b_{m-1}, \ldots, b_{m-i+1}$ and the residue of $S_{i}$ $(\bmod i)$. If the integral polynomials $g$ and $\widetilde{g}$ are distinct then we can take the minimal $i$ such that $S_{i} \neq \widetilde{S}_{i}$. Then $S_{i} \equiv \widetilde{S}_{i}(\bmod i)$ and, hence,

$$
\exists i \quad\left|S_{i}-\widetilde{S}_{i}\right| \geq i,
$$

or

$$
\exists i \quad\left|\sum_{v \in V} v^{i}+\sum_{w \in W} w^{i}-\sum_{v \in \widetilde{V}} v^{i}-\sum_{w \in \widetilde{W}} w^{i}\right| \geq i
$$

Let

$$
\begin{gathered}
W=\left\{w_{1}, \ldots, w_{m-k n}\right\}, \quad \widetilde{W}=\left\{\widetilde{w}_{1}, \ldots, \widetilde{w}_{m-k n}\right\}, \\
w_{j}=\widetilde{w}_{j}=0 \quad(m-k n<j \leq m) .
\end{gathered}
$$

Then we can deduce from (3.10) and (3.11) that

$$
\exists i \quad\left|\Re \sum_{j=1}^{m} w_{j}^{i}-\Re \sum_{j=1}^{m} \widetilde{w}_{j}^{i}\right|>i / 2 .
$$

Now we can apply Lemma 2.5: there are at most $\exp \left(m^{0.95}\right)$ polynomials $g \in \mathcal{D}(U, m, l, k)$ possessing any prescribed collection of values

$$
\left(n=\psi(g), \psi_{1}(g), \ldots, \psi_{n}(g), \Psi_{1}(g), \ldots, \Psi_{n}(g), \varphi_{1}(g), \ldots, \varphi_{n}(g)\right) .
$$

Finally, by (3.7), we find

$$
{ }^{\#} \mathcal{D}(U, m, l, k) \leq \exp \left(m^{0.95}\right) N \leq \exp \left(m^{0.95}\right) C^{U / k}(C l / k)^{C U / \log m} .
$$

This completes the proof of Lemma 3.1.

4. Proof of Theorem 2. Let $n$ be a positive integer and $\zeta$ a primitive $n$th root of unity. Then the polynomial

$$
Q_{n}(z)=\prod_{\substack{j=1 \\ \operatorname{gcd}(j, n)=1}}^{n}\left(z-\zeta^{j}\right)
$$

is called the $n$th cyclotomic polynomial [6, p. 64]. Note that $\operatorname{deg} Q_{n}=\varphi(n)$ where $\varphi$ is the Euler totient function.

Denote by $g$ an integral irreducible polynomial of positive degree $\leq m_{1}$. Let $N$ be the number of polynomials $f \in \mathcal{P}_{d}$ divisible by at least one such $g$. Recall that $m_{2}=\left[\sqrt{d} /(\log d)^{2}\right]$. Then

$$
N \leq N_{1}+N_{2}+N_{3}+N_{4}
$$

where

- $N_{1}$ is the number of $f \in \mathcal{P}_{d}$ divisible by at least one cyclotomic $g$ with $\operatorname{deg} g \leq m_{2}$, 
- $N_{2}$ is the number of $f \in \mathcal{P}_{d}$ divisible by at least one noncyclotomic $g$ with $\operatorname{deg} g \leq m_{2}$,

- $N_{3}$ is the number of $f \in \mathcal{P}_{d}$ divisible by at least one $g$ with $\operatorname{deg} g>m_{2}$ and $\log M(g) \leq c$,

- $N_{4}$ is the number of $f \in \mathcal{P}_{d}$ divisible by at least one $g$ with $m_{2}<$ $\operatorname{deg} g \leq m_{1}$ and $\log M(g)>c$.

Note that all the large constants $C$ can be effectively evaluated. Considering the values of all these constants fixed, we can choose the constant $c$ in the definitions of $N_{3}$ and $N_{4}$ and the constant $c$ in the definition of $m_{1}$ small enough to guarantee the validity of all the forthcoming inequalities including $C$ and $c$.

We have already estimated $N_{2}$ in Section 1 :

$$
N_{2}=o\left(2^{d} / \sqrt{d}\right) \text {. }
$$

It remains to give upper bounds for $N_{1}, N_{3}$, and $N_{4}$.

Clearly, no $f \in \mathcal{P}_{d}$ is divisible by $Q_{1}(z)=z-1$. For $n \geq 2$ denote by $N_{1, n}$ the number of $f \in \mathcal{P}_{d}$ divisible by $Q_{n}(z)$. Suppose that the polynomial

$$
f(z)=\sum_{j=0}^{d} a_{j} z^{j}
$$

is divisible by $Q_{n}$. Let

$$
h(z)=\sum_{j=0}^{n-1} A_{j} z^{j},
$$

where

$$
A_{j}=\sum_{k \equiv j(\bmod n)} a_{k}
$$

The polynomials $f$ and $h$ are congruent $\bmod \left(z^{n}-1\right)$. Therefore, $h$ is divisible by $Q_{n}(z)$. Let $l=\varphi(n)=\operatorname{deg} Q_{n}$. It is well known that $n \leq$ $C l \log \log (l+2)$ [8, Chapter 1, Theorem 5.1]. The divisibility of $h$ by $Q_{n}$ determines the coefficients $A_{j}(0 \leq j<l)$ of $h$ if its other coefficients are given. This means that for fixed $a_{k}(k \equiv j(\bmod n), l \leq j<n)$ all sums (4.3) for $0 \leq j<l$ are determined. For any such $j$ the proportion of vectors $\left(a_{j}, a_{j+n}, \ldots, a_{j+[(d-j) / n] n}\right)$ satisfying (4.3) among all 0,1 -vectors is at most $C \sqrt{n / d}$. We will use the fact that $C \sqrt{n / d} \leq C \sqrt{2 C m_{2}\left(\log \log m_{2}\right) / d} \leq$ $1 / d^{1 / 4}$. Therefore,

$$
N_{1,2} \leq(C \sqrt{2 / d}) 2^{d}, \quad N_{1, n} \leq 2^{d} / d^{l / 4}, \quad l=\varphi(n)>1,
$$


and

$$
\begin{aligned}
N_{1} & \leq 2^{d}\left(2 C / \sqrt{d}+\sum_{l=2}^{m_{2}} \#\{n: \varphi(n)=l\} / d^{l / 4}\right) \\
& \leq 2^{d}\left(2 C / \sqrt{d}+\sum_{l=2}^{m_{2}} C l(\log \log (l+2)) / d^{l / 4}\right) \leq 3 C \cdot 2^{d} / \sqrt{d}
\end{aligned}
$$

To estimate $N_{3}$, we use the following simple

Lemma 4.1. For any irreducible polynomial $g$ with $\operatorname{deg} g=m$, there are at most $2^{d-1-m}$ polynomials $f \in \mathcal{P}_{d}$ divisible by $g$.

Pr o of. There are $2^{d-1-m}$ choices of coefficients $a_{j}(j=m+1, \ldots, d-1)$ of a polynomial $f \in \mathcal{P}_{d}$. If these coefficients are fixed, the other coefficients are determined by the condition of divisibility of $f$ by $g$. The lemma is proved.

Applying Lemma 3.1 for $U=\mathrm{cm}, k=l=1$, we find that there are at most $1.5^{m}$ distinct polynomials $g$ with $\operatorname{deg} g=m$ and $\log M(g) \leq c$ (provided that $c$ is small enough). Hence,

$$
N_{3} \leq 2^{d-1} \sum_{m=m_{2}+1}^{\infty} 1.5^{m} \cdot 2^{-m}=o\left(2^{d} / \sqrt{d}\right) .
$$

REMARK. We have not yet used the restriction $\operatorname{deg} g \leq m_{1}$. Thus, we have proved that the number of polynomials $f \in \mathcal{P}_{d}$ having at least one nontrivial integral divisor $g$ with $\log M(g) \leq c$ (in particular, cyclotomic $g$ ) does not exceed $C 2^{d} / \sqrt{d}$.

The most delicate part of the proof is the estimation of $N_{4}$. We will deal with an irreducible polynomial $g$, dividing at least one polynomial $f \in \mathcal{P}_{d}$, such that $m_{2}<\operatorname{deg} g=m \leq m_{1}$ and $\log M(g)>c$. Applying (1.1) to $f$ we have

$$
M(g) \leq M(f) \leq d+1 .
$$

We use the notation of Section 3. Let

$$
l(g)=\min \left\{l \in \mathbb{Z}_{+}:(l+1) \log M(g) / k_{l+1}>\log (d+1)\right\} .
$$

By (4.6), $l \geq 1$. We need the upper estimate of $l(g)$.

LEMMA 4.2. The number $l=l(g)$ satisfies

$$
(2 C \log (d+1) / \log M(g))^{C m \log M(g) / \log m} \leq 1.05^{d / l},
$$

where $C$ is the same constant as in the statement of Lemma 3.1.

(We may consider $C>1$.) 
Proof. Set $A=2 C \log (d+1) / \log M(g)$,

$$
l_{1}=\frac{d(\log 1.05) \log m}{C m(\log M(g)) \log A} .
$$

( $A>2$ by (4.6).) We have

$(\log M(g)) \log A \leq 2 C \log (d+1) / e \leq 2 C \log m_{2} \leq 2 C \log m$,

$$
d(\log m) / m \geq\left(d / m_{1}\right) \log m
$$

and, consequently,

$$
l_{1} \geq \frac{d(\log 1.05) \log m}{2 C^{2} m \log m} \geq d(\log 1.05) /\left(2 C^{2} m_{1}\right)>6 \log m
$$

(for an appropriate choice of a small constant $c$ in the definition of $m_{1}$ ). The lemma asserts that $l(g) \leq l_{1}$. Assume the opposite. Then for any $l \in\left(l_{1} / 2, l_{1}\right]$ we have

$$
\begin{aligned}
k_{l} & \geq l \log M(g) / \log (d+1)>l_{1} \log M(g) /(2 \log (d+1)) \\
& =\frac{d(\log 1.05) \log m}{2 C m(\log (d+1)) \log A} \\
& \geq \frac{d(\log 1.05) \log m_{1}}{2 C m_{1}(\log (d+1)) \log (2 C \log (d+1) / c)} \\
& >(\log d)^{1 / 2}>(\log m)^{1 / 2}
\end{aligned}
$$

Let $p$ run over primes in $\left(l_{1} / 2, l_{1}\right]$; as $l_{1}$ is sufficiently large, there are at least $0.4 l_{1} / \log l_{1}$ such primes. By the property (iii) of Lemma 3 from [3] and (4.8) for $L=\prod_{p} p$ we have

$$
k_{L} \geq \prod_{p} k_{p} \geq(\log m)^{0.2 l_{1} / \log l_{1}}
$$

and substitution of (4.7) gives

$$
k_{L} \geq(\log m)^{1.2 \log m / \log (6 \log m)}>m .
$$

The last inequality is impossible. Thus, our assumption was not correct, and Lemma 4.2 is proved.

For a nonnegative number $U$ such that $2 c m \leq U \leq 2 m \log (d+1)$ and positive integers $k, l, m$ with $m_{1}<m \leq m_{2}$, we denote by $\mathcal{D}(U, m)$ the set of irreducible polynomials $g$ of degree $m$ such that $U /(2 m)<\log M(g) \leq U / m$, and by $\mathcal{D}(U, m, l)$ the set of polynomials $g \in \mathcal{D}(U, m)$ such that $l(g)=l$. By the definition of $l(g)$,

$$
k_{l} \geq \frac{l \log M(g)}{\log (d+1)} \geq \frac{l U}{2 m \log (d+1)}=K .
$$

Lemma 3.1 gives the estimate of the number of polynomials in $\mathcal{D}(U, m, l)$ : 


$$
\begin{aligned}
& { }^{\#} \mathcal{D}(U, m, l) \\
& \quad \leq \sum_{k=K}^{l}{ }^{\#} \mathcal{D}(U, m, l, k) \leq \sum_{k=K}^{l} \exp \left(m^{0.95}\right) C^{U / k}(C l / k)^{C U / \log m} \\
& \quad \leq l \exp \left(m^{0.95}\right) C^{U / K}(C l / K)^{C U / \log m}
\end{aligned}
$$

We have

$$
\begin{gathered}
C^{U / K}=C^{2 m \log (d+1) / l} \leq C^{2 m_{1} \log (d+1) / l} \leq 1.05^{d / l}, \\
(C l / K)^{C U / \log m} \leq\left(\frac{2 C m \log (d+1)}{U}\right)^{C U / \log m} \\
\leq\left(\frac{2 C m \log (d+1)}{m \log M(g)}\right)^{2 C m \log M(g) / \log m},
\end{gathered}
$$

and, hence, by Lemma 4.2,

$$
(C l / K)^{C U / \log m} \leq 1.05^{2 d / l} .
$$

Substituting (4.10) and (4.11) into (4.9), we get

$$
\begin{aligned}
{ }^{\#} \mathcal{D}(U, m, l) & \leq \sum_{k=K}^{l}{ }^{\#} \mathcal{D}(U, m, l, k) \leq l \exp \left(m^{0.95}\right) 1.05^{3 d / l} \\
& \leq \exp \left(m^{0.96}\right) 1.05^{3 d / l} \leq \exp \left(2 m^{0.96}\right)+1.05^{6 d / l}
\end{aligned}
$$

Repeating the same arguments as in Section 1, we now show that for any $g \in \mathcal{D}(U, m, l)$ the condition of divisibility of $f(z)=\sum_{j=0}^{d} a_{j} z^{j} \in \mathcal{P}_{d}$ by $g$ uniquely determines $f$ by its coefficients $a_{j}$ with $j \not \equiv 0(\bmod l+1)$. Indeed, let $z_{1}, \ldots, z_{m}$ be the zeros of $g$, and $G$ be the minimal polynomial for $z_{1}^{l+1}$. Then $G^{k}(w)=\prod_{j=1}^{m}\left(w-z_{j}^{l+1}\right)$ where $k=k_{l+1}$. By the definition of $l(g)$, we get

$$
M(G)=M(g)^{(l+1) / k}>d+1 .
$$

Suppose that $g(z)$ divides two distinct polynomials $f_{1}$ and $f_{2}$ from $\mathcal{P}_{d}$ such that

$$
f_{1}(z)-f_{2}(z)=\sum_{j=0}^{[d / p]} a_{j} z^{j(l+1)}=h\left(z^{l+1}\right) .
$$

Clearly, all the coefficients of the polynomial $h$ are $0, \pm 1$. By $(1.1), M(h) \leq$ $d+1$. On the other hand, $h$ is divisible by $G$, and (4.13) entails $M(h)>d+1$. This contradiction shows that our supposition cannot occur. Hence,

$$
\#\left\{f \in \mathcal{P}_{d}: g \mid f\right\}<2^{d} / 2^{d /(l+1)}<2^{d} / 1.41^{d / l} .
$$

Combining this estimate with Lemma 4.1 and using (4.12) and the inequality 
$1.05^{7}<1.41$, we get

$$
\begin{aligned}
\#\left\{f \in \mathcal{P}_{d}:\right. & \exists g \in \mathcal{D}(U, m) g \mid f\} \\
& <2^{d} \sum_{l}\left(\exp \left(2 m^{0.96}\right)+1.05^{6 d / l}\right) \min \left(2^{-m}, 1.41^{-d / l}\right) \\
& \leq 2^{d}\left(\sum_{l} \exp \left(2 m^{0.96}\right) 2^{-m}+\sum_{l} 1.05^{-d / l}\right),
\end{aligned}
$$

where the sum is taken over $l$ for which there exists at least one polynomial $g \in \mathcal{D}(U, m, l)$ dividing some polynomial $f \in \mathcal{P}_{d}$. By Lemma 4.2, any such $l$ satisfies

$$
1.05^{-d / l} \leq C^{-c C m / \log m} .
$$

Hence, it follows from (4.14) that

$$
\begin{aligned}
\#\left\{f \in \mathcal{P}_{d}: \exists g \in \mathcal{D}(U,\right. & m) g \mid f\} \\
\leq & 2^{d} m\left(\exp \left(2 m^{0.96}\right) 2^{-m}+C^{-c C m / \log m}\right) \\
& <2^{d} \exp (-\sqrt{m}) .
\end{aligned}
$$

Set $U_{j, m}=\mathrm{cm} \cdot 2^{j}$ and note that if $f \in \mathcal{P}_{d}$ is divisible by some polynomial $g$ with $m_{2}<m=\operatorname{deg} g \leq m_{1}$ and $\log M(g)>c$, then $g \in \mathcal{D}\left(U_{j, m}, m\right)$ for some $j$ with $1 \leq j \leq J=1+[\log (d+1) / c]$. Thus, from (4.15) we get

$$
\begin{aligned}
N_{4} & <2^{d} \sum_{j=1}^{J} \sum_{m=m_{2}+1}^{m_{1}} \exp (-\sqrt{m}) \\
& <C 2^{d} m_{1} \exp \left(-\sqrt{m_{2}}\right) \log (d+1)=o\left(2^{d} / \sqrt{d}\right) .
\end{aligned}
$$

The substitution of (4.2), (4.4), (4.5), and (4.16) into (4.1) completes the proof of Theorem 2.

As we have seen in Section 1, Theorem 1 is a corollary of Theorem 2.

\section{References}

[1] I. Bárány and Z. Füredi, Computing the volume is difficult, Discrete Comput. Geom. 2 (1987), 319-326.

[2] J. Brillhart, M. Filaseta and A. M. Odlyzko, On an irreducibility theorem of A. Cohn, Canad. J. Math. 33 (1981), 1055-1059.

[3] E. Dobrowolski, On a question of Lehmer and the number of irreducible factors of a polynomial, Acta Arith. 34 (1979), 391-401.

[4] M. Filaseta, Irreducibility criteria for polynomials with nonnegative coefficients, Canad. J. Math. 40 (1988), 339-351.

[5] L. Kronecker, Zwei Sätze über Gleichungen mit ganzzahligen Coefficienten, J. Reine Angew. Math. 53 (1857), 133-175.

[6] R. Lidl and H. Niederreiter, Finite Fields, Addison-Wesley, 1983. 
[7] A. M. Odlyzko and B. Poonen, Zeros of polynomials with 0,1 coefficients, Enseign. Math. 39 (1993), 317-348.

[8] K. Prachar, Primzahlverteilung, Springer, Berlin, 1957.

[9] V. Tikhomirov, Some Problems of Approximation Theory, Moscow State University, Moscow, 1976 (in Russian).

[10] E. C. Titchmarsh, The Theory of Functions, 2nd ed., Oxford Univ. Press, 1939.

Department of Mechanics and Mathematics

Moscow State University

Moscow, 119899, Russia

E-mail: kon@nw.math.msu.su

Received on 28.5.1997

and in revised form on 28.11.1998 